\title{
O uso de Robótica para aprendizado de Programação integrando alunos de Educação Básica e Ensino Superior
}

\author{
Luemy Avila $^{1,2}$, Flavia Cristina Bernardini ${ }^{3}$, Patrick Moratori ${ }^{3}$ \\ ${ }^{1}$ Faculdade de Educação \\ Universidade Estadual do Rio de Janeiro - UERJ \\ Rio de Janeiro, RJ, Brasil \\ ${ }^{2}$ Secretaria de Educação de Macaé \\ Prefeitura Municipal de Macaé \\ Macaé, RJ, Brasil \\ ${ }^{3}$ Laboratório de Inovação no Desenvolvimento de Sistemas - LabIDeS \\ Instituto de Ciência e Tecnologia - ICT \\ Universidade Federal Fluminense - UFF, Campus Rio das Ostras \\ Rio das Ostras, RJ, Brasil \\ luemyavila@gmail.com, \{fcbernardini,pmoratori\}@id.uff.br
}

\begin{abstract}
Nowadays, it is been hard to keep both engagement and attention of students during their learning process due to the broad information access provided by the internet. Moreover, students in general have been having difficulties in logical reasoning, concepts abstraction and problem solving. On the other hand, Computer Science undergraduate students have been reporting a lack of motivation during their initial academic terms, because it is difficult to them to understand applications of their learned knowledge. Consequently, the use of robotic programming allow interactions between students from basic education and undergraduate courses, which allow a learning process through discussions and collaboration between them in performing tasks using technology tools. In this context, a case study is presented in this article. A project was carried out to join local students from undergraduate courses and basic education aiming to learn robotic programming. The results indicate positive effects on participants, improving their self-confidence, abstraction skills and problem solving capability.
\end{abstract}

Resumo. Atualmente, há uma dificuldade em manter o interesse e a atenção dos estudantes no seu aprendizado devido ao amplo acesso à informação trazido pela internet. Ainda, os alunos em geral apresentam dificuldade em raciocínio lógico, abstração de conceitos e resolução de problemas. Por outro lado, alunos de graduação em Ciência da Computação apresentam falta de motivação nos períodos iniciais, já que a aplicação direta dos conhecimentos obtidos na universidade muitas vezes não é percebido. Daí, a interação entre alunos com diferentes níveis de formação para programação de robôs pode permitir um processo de aprendizado por meio de discussões e colaborações na realização de tarefas, utilizando ferramentas tecnológicas. Nesse contexto, um estudo de caso é apresentado neste trabalho. Foi executado um projeto que visava 
uma ampla interação entre alunos de graduação e de escolas do município local para aprendizado de programação em robótica. Os resultados indicaram efeitos positivos nos participantes, onde se observou melhoria nos quesitos autoconfiança, abstração de conceitos e resolução de problemas.

\section{Introdução}

Nas últimas décadas a educação tem estado carente de inovações para lidar com os desafios da contemporaneidade, existindo um descompasso entre os avanços tecnológicos e a realidade escolar. Observa-se que as gerações atuais convivem cotidianamente com diversos aparatos tecnológicos e um amplo acesso à informação para realizar suas atividades diárias, seja para ler um livro digital ou para comprar um produto pela internet. Porém, tanto o ambiente quanto a estrutura e as metodologias aplicadas nas escolas nem sempre estão alinhados à esta modernidade [MORIN 1996, DEMO 1998, FRAWLEY 2000], principalmente quando se considera a realidade das instituições públicas brasileiras. Consequentemente, existem demandas por estratégias inovadoras que permitam inovar, motivar e manter o interesse deste novo perfil de estudantes.

Para que um aluno possa ter uma posição ativa no processo ensino-aprendizado é preciso que ele seja capaz de abstrair problemas do seu dia-a-dia, a partir do desenvolvimento de seu raciocínio lógico. Neste contexto, a utilização de tecnologias na área educacional tem se tornado uma questão de estado em diversos países da Europa e na América do Norte, onde a utilização de ensino de programação e robótica aplicados à problemas do mundo real tem permitido melhorias em abstrações, atraindo e motivando estudantes em diversos contextos [PEREIRA 2010, BENITTI et al. 2009, AGUIAR et al. 2015, MARINS 1999, RIBEIRO et al. 2011].

As parcerias entre universidades e escolas permitem ações nesse sentido, onde alunos com diferentes níveis de formação podem ser integrados para um aprendizado por discussões e colaborações na realização de tarefas, tornando possível: (i) atrair e motivar estudantes para resolver problemas em diversos contextos; (ii) aproximar alunos da Educação Básica em relação à universidade, conhecendo melhor a realidade e a potencialidade de cursos de graduação; (iii) motivar alunos do Ensino Superior à contribuir com a sociedade, vendo aplicabilidade de seus conhecimentos; e (iv) diminuição de evasões escolares e universitárias. Tais iniciativas são relevantes no âmbitos acadêmico-profissional, pois existe uma relação direta entre os setores de tecnologia e o desenvolvimento de ações inovadoras. Porém, um número decrescente de profissionais tem estado disponível no mercado, principalmente pela elevada desistência de alunos em cursos de graduação em áreas relacionadas, como Ciência da Computação e Engenharias [da SILVA et al. 2013].

Este artigo apresenta um estudo de caso de uma atividade de extensão. Trata-se da execução de um projeto durante o ano de 2015 que visava a integração entre alunos da graduação em Ciência da Computação da Universidade Federal Fluminense (UFF) Campus Rio das Ostras e estudantes da Educação Básica (ensino fundamental e médio) de escolas locais da cidade. A ideia principal é utilizar a robótica no ensino de programação como uma estratégia inovadora na relação educação-tecnologia, observando os fatores motivacionais. A iniciativa do projeto de robótica no Campus Universitário envolve alunos do ensino fundamental, médio e superior, além de seus respectivos docentes. Para a execução deste projeto, é apresentada uma metodologia, identificando as atividades 
realizadas bem como a sua avaliação à posteriori pelos participantes.

As seções subsequentes possuem a seguinte organização: a Seção 2 apresenta a motivação e os trabalhos relacionados ao aprendizado de programação pela robótica; a Seção 3 descreve a metodologia aplicada na integração Universidade-Escola no uso da tecnologia associada ao ensino; a Seção 4 discute os resultados obtidos; e por fim, a Seção 5 apresenta as conclusões e trabalhos futuros.

\section{O aprendizado de programação na Educação Básica e no Ensino Superior}

Atualmente existem demandas para o uso de tecnologias no processo educacional. Iniciativas como o CODE no ensino de programação [CODE 2015] conta com o apoio de diversas empresas e importantes personalidades da atualidade, como Google, Microsoft, Salesforce, Omidyar Network, Reid Hoffman, Mark Zuckerberg and Priscilla Chan, Bill Gates, Drew Houston, Ballmer Family Giving, Ali Partovi and Hadi Partovi, Sean N. Parker Foundation, Diane Tang and Ben Smith, John and Ann Doerr, Quadrivium Foundation, dentre outros. Projetos como "Scratch" [MIT Media Lab 2015], do MIT, e "Eu posso programar" [MICROSOFT 2015], da Microsoft, reforçam essa perspectiva considerando a importância da introdução de conceitos de programação a jovens estudantes, no intuito de permitir uma melhor compreensão de abstrações presentes no mundo real. Tal fato permite compreender o grau de importância que a programação e conceitos associados à área de Ciência da Computação podem impactar no cenário de uma nova concepção do uso e geração de tecnologias e inovações, principalmente quando ensinados em séries iniciais da Educação Básica. Entretanto, um estudo divulgado pela SOFTEX aponta que o Brasil pode chegar em 2020 com um déficit de mão de obra qualificada em TI de 408 mil profissionais. Tal estimativa, que destaca uma posição pessimista, mostra que essa escassez se tornará um problema educacional caso o país não reforce programas para reverter tal situação [COMPUTERWORLD. 2013]. Tal situação é recorrente, pois um estudo da IDC, encomendado pela Cisco em abril de 2013, já havia destacado que até 2015 a demanda na América Latina por profissionais de TI especializados seria 35\% maior que a força de trabalho disponível no mercado, chegando a um déficit de aproximadamente 296 mil trabalhadores [NOTÍCIAS DA CISCO. 2013].

Neste sentido, a robótica pode contribuir para a motivação de alunos tanto da Educação Básica quanto do Ensino Superior, pois a aplicação de tecnologias a problemas do mundo real viabiliza as seguintes características [CASTILHO 2002, DEMO 1998, MAISONNETTE 1999, FRANCO 1998]: (i) promover a qualificação profissional do cidadão; (ii) democratizar o acesso aos conhecimentos produzidos para a melhoria da qualidade de vida; (iii) promover a cidadania e valores democráticos aos diferentes atores sociais que se envolvem de forma direta e indireta nas ações; (iv) enriquecer a proposta educacional escolar, implantado ou fortalecendo a aprendizagem pela resolução de problemas, de forma contextualizada, além de desenvolver os valores estéticos, políticos e éticos, organizados sob as premissas de sensibilidade, igualdade e identidade; (v) promover a construção flexível dos saberes com foco na mediação da aprendizagem; e (vi) incentivar que jovens em diferentes faixas etárias se enveredem por áreas relacionadas ao desenvolvimento da Ciência e Tecnologia.

Em diversos trabalhos na literatura são apresentados estudos nos quais se utiliza a programação associada à robótica com intuitos motivacionais. Em [PEREIRA 2010] 
é apresentado um trabalho sobre o uso do Lego Mindstorms como uma ferramenta de robótica para o aprendizado de conceitos básicos de computação em uma escola da Zona Rural de Catalão, GO. Em [BENITTI et al. 2009] são apresentados os resultados de um experimento do uso de robôs no ensino médio em diferentes disciplinas do currículo escolar. Em [MARINS 1999], destaca-se a importância da robótica na escola, descrevendo que entre as dez escolas curitibanas que obtiveram as melhores notas no ENEM em 2012, sete oferecem a robótica como disciplina extracurricular. Um representante destas escolas afirma que tal disciplina vem sendo incluída na grade curricular por ser uma atividade lúdica e que, quando o aluno se sente livre, ele aprende mais facilmente. Em [AGUIAR et al. 2015] é apresentada uma proposta de uso de programação em robótica para ensinar a lógica de programação de uma forma indutiva, ou seja, construção de inferências à partir de casos observados. O diferencial da metodologia descrita neste artigo, em contraponto aos trabalhos já disponibilizados na literatura, se dá pela integração entre universidade e escola, identificando os benefícios proporcionados por esta parceria. Em [RIBEIRO et al. 2011], foi realizado um estudo do uso de robótica em disciplinas de programação básica no primeiro período de cursos de graduação, que permitiu observar que o uso da robótica motiva os alunos iniciantes na graduação na programação, bem como facilita o desenvolvimento do raciocínio lógico.

\section{Uma metodologia para programação de robôs na integração entre Universidade e Escola}

A estratégia principal aplicada no aprendizado de programação utilizando robótica consiste na criação de oficinas que permitem aos alunos debater conceitos relacionados ao seu dia-a-dia. Desta maneira, a sistematização de informações se torna necessária, viabilizando a aquisição de novos conhecimentos durante o processo de construção de um robô. Para se atingir esse objetivo, os envolvidos devem percorrer diversas etapas para viabilizar o desenvolvimento do pensamento criativo, como: (1) a elaboração visual de protótipos a partir de desenhos e gráficos; (2) a utilização do pensamento sistemático que define sequências de ações para resolução de problemas; (3) o uso da escrita para documentar, de maneira clara e objetiva, os passos envolvidos nos projetos realizados; (4) a prática do raciocínio lógico para transpor sistematizações em códigos de programação nos dispositivos que estão sendo construídos; e (5) o processo de pesquisa para a identificação de demandas, onde soluções e facilitadores possam ser propostos. Essas etapas propiciam aos estudantes uma maior maturidade para lidar com desafios da vida, pois os mesmos são instigados à ter uma postura crítica e analítica frente a problemas.

O objetivo principal deste projeto é preparar estudantes da Educação Básica para o desenvolvimento de suas habilidade cognitivas, além de viabilizar a capacitação dos acadêmicos extensionistas. Além da geração de um campo de estágio lúdico para os universitários, existe o estímulo pelo estudo de ciências entre os beneficiados pelo projeto. Desta maneira, jovens passam a considerar a universidade como uma opção viável e favorável à sua formação como indivíduo, possibilitando impactos positivos para a elevação de níveis socioeconômicos e potencializando a qualidade de vida no município. Vale ressaltar que Rio da Ostras está em uma área estratégica na produção de petróleo no país, fazendo parte de um nicho de grande utilização profissional de robôs na indústria.

Para que o projeto atinja um de seus objetivos, que é despertar o interesse nas áreas de Ciências Exatas e Tecnológicas, mais especificamente em Computação e Engenharia, 
para alunos da rede pública, é proposta a criação de oficinas direcionadas aos alunos para construção de conceitos e aplicabilidade do conhecimento para a construção de um robô. Tais oficinas podem evidenciar o objetivo de solucionar problemas com ideias inovadoras a partir do levantamento da demanda social, tornando a engenharia e a computação algo lúdico, mais palpável que os cálculos matemáticos, que podem ser assustadores para iniciantes. Para se atingir esse objetivo, os alunos percorrem diversas etapas que possibilitam o desenvolvimento do pensamento criativo, que envolvem: (1) a elaboração de desenhos para protótipos; (2) a sistematização do pensamento para a resolução de problemas; (3) a escrita para documentar, de modo claro e sólido, os passos dos projetos de construção de robôs; (4) o uso do raciocínio lógico para programar os dispositivos construídos; e (v) o processo de pesquisa para levantamento das demandas que necessitam solucionar e ou facilitar o desenvolvimento do robô. Esse desenvolvimento propicia maturidade e uma postura crítica e analítica para os desafios da vida. Para uma execução inicial do projeto, é proposto que o grupo seja composto por até 20 alunos dos Ensinos Fundamental e Médio da cidade de Rio das Ostras. O grupo pode utilizar kits de robótica para trabalhos educacionais. Kits comumente utilizados são os da linha Lego ${ }^{\circledR}$ Mindstorms, utilizados para a execução deste projeto. Esses kits são compostos por (1) blocos de montagem padrão Lego; (2) peças móveis como engrenagens, roldanas, eixos e rodas; (3) sensores de toque e ultrassônico, e (4) módulo principal, o qual é um mini computador programável. Uma IDE (Integrated Development Environment) para programação em blocos acompanha o kit educacional. Essas peças podem ser combinadas de diversas formas, de acordo com a criatividade dos alunos, para a formação de diferentes dispositivos robóticos. Deve ser observado que os kits Lego ${ }^{\circledR}$ Mindstorms não é um simples brinquedo. Tais kits foram concebidos para que estudantes pensem sobre padrões e estruturas não apenas de robótica, mas também de programação e resolução de problemas de forma mais geral. Como o projeto visa levar o aprendizado de programação e o uso do raciocínio lógico para soluções de problemas a alunos de Educação Básica, esses alunos devem (1) pesquisar e desenvolver programas utilizando programação em robótica utilizando os kits; e (2) desenvolver robôs e estruturas que podem ser trabalhadas para a resolução de algumas tarefas que possam ser aplicadas no cotidiano.

Durante os encontros são elaborados alguns esquemas e táticas para realização de tarefas, onde cada aluno participa integralmente do processo, ou seja, desde a montagem de robôs até sua programação e cálculos necessários para construir soluções. Para realizar tais missões, os alunos recebem orientações dos monitores de graduação para desenvolverem tanto soluções lógicas quanto táticas de programação. Este processo é facilitado pelo uso da IDE que acompanha o Lego Mindstorm, na qual as soluções são desenvolvidas a partir de sequências de blocos programáveis, permitindo, de maneira intuitiva, a implementação dos mais diversos comportamentos desejados. Por exemplo, para que o robô ande para alguma direção, é utilizado o bloco de ação que envia um comando para os motores responsáveis pela locomoção. Similarmente, pode-se implementar que o robô apenas realize uma determinada tarefa após a detecção de um determinado obstáculo, ou seja, terá que aguardar uma resposta de algum dos sensores utilizados, seja ele de toque ou ultrassônico, para que a ação seja desempenhada. Dessa maneira, os alunos podem acompanhar o desenvolvimento e execução de tarefas, partindo de algo conceitual e teórico para soluções práticas. Com isso, várias alternativas são exploradas para a resolução de problemas específicos. 
Além do acompanhamento durante a execução do projeto, ao final é indicada uma reunião com todos os participantes do projeto, ou a aplicação de questionários, para identificar os pontos fortes e fracos durante a execução. Também, atividades pouco trabalhadas por alguns alunos podem ser variadas no ano subsequente para outros conhecimentos serem trabalhados, retirando os alunos de suas zonas de conforto.

\section{Resultados}

Para compor a turma para participação no projeto, foram visitadas duas escolas, uma de ensino fundamental do município, e outra de ensino médio. A execução do projeto teve duração de março a dezembro de 2015. Participaram ao longo do projeto onze alunos das duas escolas e cinco alunos de graduação. É importante observar que alguns alunos das escolas desistiram do projeto no decorrer da execução, quanto outros entraram no meio do ano. Como não havia notas associadas ne certificado de participação, não havia a obrigatoriedade da participação. Um dos critérios para participação era a oportunidade de trabalhar com desenvolvimento de programas e resolução de problemas com robótica para participar tanto de torneios quanto olimpíadas de programação de robôs. Para o desenvolvimento dos robôs, foram utilizados três kits Lego Mindstorms - um kit NXT e dois kits EV3. Observou-se que os conhecimentos adquiridos durante as reuniões faziam com que os alunos ficassem mais confiantes em suas habilidades interpessoais e intelectuais, e consequentemente, mais entusiasmados com o projeto.

Em pouco tempo de projeto, notou-se grande empenho nos participantes para participar de eventos relacionados, vista a formação de duas equipes para participar das Etapa Regional e Estadual das Olimpíadas Brasileiras de Robótica - OBR e uma equipe para participar da competição First Lego League - FLL. As equipes que participaram da OBR regional, em junho de 2015, ficaram em segundo e décimo primeiro lugar na competição de desenvolvimento de tarefas pelo robô. Já na etapa estadual, em outubro de 2015, uma das equipes conquistou o prêmio de robô com o melhor design. Para a etapa regional da FLL, os alunos tinham que fazer uma pesquisa sobre a temática da competição. Escolheram o tema lixo eletrônico. Foi construído um painel com exemplos de lixo eletrônico, um folder de explicação dos problemas desse tipo de lixo, e um jogo programado em Scratch também sobre essa temática. Essa competição ocorrer em Novembro de 2015. O time não foi premiado premiação, mas foi fundamental a participação dos alunos no evento para perceberem a importância do trabalho em equipe, fundamental para as carreiras profissionais atualmente. Observa-se que a experiência dos alunos nesses eventos trouxeram uma maior maturidade para os alunos, deixando mais cientes de possíveis desafios que estão por vir na competição.

O entusiasmo dos alunos ficou evidente quando se observou seus comportamento nesses eventos, desde o momento da inscrição, a escolha do nome da equipe, até o começo dos treinos para a competição. Percebe-se melhorias na formas de trabalho e na interação com os outros parceiros de equipe, onde são construídos vínculos de lealdade e confiança. Em outra perspectiva, observa-se o impacto positivo observado por uma professora de Física do colégio estadual que acompanhou as atividades. A professora afirmou: "Pude perceber uma melhoria quanto ao [meu] aluno em sala de aula, uma vez que, antes dele frequentar o projeto não tinha tanta motivação no aprendizado e era muito dependente de mim para realizar os exercícios propostos em sala de aula. Depois que ele começou a ir aos encontros pude perceber que estava bem menos dependente para a execução de 
atividades e mais comunicativo com a sua turma."

Já para os alunos da graduação, pôde-se perceber que a vontade de ensinar e aprender com esses alunos é muito gratificante, pois mesmo em um projeto piloto, os alunos possuem grande liderança, dedicação e proatividade. Um dos alunos monitores do projeto, do curso de Ciência da Computação, afirmou: "O legal do projeto é que em nossas reuniões não existe a definição de aluno e professor, todos estamos ali para ensinar e aprender. Eu mesmo aprendi muito com um dos alunos do ciclo básico de apenas 15 anos e que tem uma enorme facilidade com a programação dos blocos em Lego". Outro aluno da graduação também considerou: "[...] tem sido muito gratificante e proveitoso participar deste processo, pois desenvolvemos nossas habilidades de comunicação e colaboramos com a difusão do conhecimento no decorrer do estudo. Na questão de qualificação profissional, sentimos que não estamos presos a um único trabalho especifico e sim a vários desafios em paralelo, visto que durante o desenvolvimento das tarefas vão surgindo novas possibilidades de pesquisas. Neste momento, compreendemos de forma mais objetiva a importância de um projeto [como este] para a formação mais integral de um profissional em relação a um mercado de trabalho cada vez mais competitivo. Conseguimos melhorar o nosso desempenho acadêmico, já que aprendemos a ser mais comunicativos, trabalhamos em equipe e resolvemos problemas proativamente."

Ao final da execução do projeto, foi feita uma chamada para uma reunião com todos os membros participantes do projeto. Compareceram à reunião seis alunos das escolas, denominados $e_{1}, \ldots, e_{6}$, e três alunos da graduação, denominados $g_{1}, \ldots, g_{3}$. Foi ouvido um depoimento de cada aluno, cujo resumo está descrito a seguir:

- $e_{1}$ (participou de todas as competições do time), que observou-se executar um papel de líder no time, disse que aprendeu os valores de trabalho em equipe, e que passou a aplicar tais valores na escola, tendo bons resultados nas suas relações interpessoais;

- $e_{2}$ (participou da FLL) ficou interessado no projeto por ter gosto pelas disciplinas de matemática e física, mas também gostou de programação e da participação na FLL;

- $e_{3}$ (participou da FLL) gostou do projeto por fazer novos amigos, mas também sentiu que tinha que levar as atividades mais a sério que a própria escola, pois tinha mais interesse e motivação. Aprender lógica de programação com os alunos da graduação ajudou na programação em Scratch e no Lego;

- $e_{4}$ (participou da FLL) afirmou que gostou muito da participação no projeto e no torneio FLL. Atuou bastante na montagem do robô, o que gostou bastante, mas achou complicada a programação;

- $e_{5}$ (participou da OBR regional e estadual) disse que compôs uma equipe sozinho para participar dos eventos, o que tornou as atividades mais difícil. Ainda assim, gosta de programar, e ressaltou suas atividades realizadas para o time participar da FLL, apesar de não ter participado;

- $e_{6}$ disse que ajudou bastante nas pesquisas de materiais na temática de lixo eletrônico, pois tinha dificuldade em programar e montar o robô;

- $g_{1}$ disse que percebia os diferentes perfis entre os alunos, e tentava ser rigoroso para garantir o bom andamento do trabalho;

- $g_{2}$ observou que a equipe era grande e que ninguém se conhecia, e observou que, com a interação, subgrupos foram sendo construídos. Um dos alunos de escola 
tinha tido problemas com a equipe, e $g_{2}$ acredita que um dos motivos foi essa questão do subgrupo;

- $g_{3}$ disse que sempre chamava a atenção da equipe para construírem o robô, fazendo com que os alunos voltassem sua atenção para as atividades da competição.

Vale salientar que os alunos colaboraram entre si, passando conhecimentos para colegas, ou até mesmo absorvendo, de forma mais intuitiva, o que tinha ficado previamente com dúvidas. Assim, fica facilitado o acúmulo de conhecimentos nas tentativas de resolução dos desafios lançados em grupo. Adicionalmente, nota-se que os alunos ficam mais proativos e estimulados na busca de novas informações e, assim, vão deixando a timidez e ficando cada vez menos dependentes dos monitores da graduação.

\section{Conclusões e trabalhos futuros}

Neste trabalho, uma metodologia foi apresentada para execução de um projeto para aprendizado de robótica que visa a integração entre alunos de Educação Básica e Ensino Superior. Tal integração pretende atrair e motivar estudantes para resolver problemas em diversos contextos através de atividades em grupo, aproximando alunos da Educação Básica à Universidade e motivando alunos do Ensino Superior à contribuir com a sociedade, vendo aplicabilidade de seus conhecimentos. Na execução deste projeto, foi possível observar para alguns participantes da Educação Básica, uma melhoria na autoconfiança, no raciocínio lógico e na resolução de problemas. Para os alunos de graduação, foi possível observar também que viam a aplicabilidade de seus conhecimentos também na área da educação. Como trabalhos futuros, pretende-se ampliar a investigação do impacto deste projeto no percurso escolar dos alunos participantes do projeto.

\section{Agradecimentos}

Agradecemos à Universidade Federal Fluminense pelas bolsas concedidas para a execução deste projeto e à Odebrecht Óleo e Gás pelo apoio financeiro para participação dos alunos nas competições.

\section{Referências}

[AGUIAR et al. 2015] AGUIAR, Y., MACIEL, B., MATTOS, S., SOARES, L., and OLIVEIRA, V. (2015). Introdução à robótica e estímulo à lógica de programação no ensino básico utilizando o kit educativo lego ${ }^{\circledR}$ mindstorms. In Anais do IV Congresso Brasileiro de Informática na Educação (CBIE 2015). Disponível em http: //www.br-ie.org/pub/index.php/wie/article/view/2166.

[BENITTI et al. 2009] BENITTI, F., VAHLDICK, A., URBAN, D., KRUEGER, M., and HALMA, A. (2009). Experimentação com robótica educativa no ensino médio: ambiente, atividades e resultados. In WIE 2009. Disponível em http://www.br-ie. org/pub/index.php/wie/article/view/2166.

[CASTILHO 2002] CASTILHO, M. (2002). Robótica na educação: Com que objetivos? Dissertação de Mestrado - Universidade Federal do Rio Grande do Sul. Disponível em http://www.proinfo.gov.br/upload/biblioteca.cgd/192. pdf. Acessado em 28 de fevereiro de 2016.

[CODE 2015] CODE (2015). Code: Anybody can learn. [online]. Disponível em https : / / code. org/. Acessado em 28 de fevereiro de 2016. 
[COMPUTERWORLD. 2013] COMPUTERWORLD. (2013). Déficit de talentos de ti no brasil pode chegar a 408 mil em 2020. [online]. Disponível em http://computerworld.com.br. Acessado em 06 de março de 2016.

[da SILVA et al. 2013] da SILVA, H. M., de ALEMIDA, L. M., BATISTA, I. D. S., and GORGÔNIO, F. L. (2013). Uma reflexão sobre o crescente desinteresse e a constante evasão em cursos de Computação e Informática. In Proceedings of International Conference on Engineering and Technology Education, volume 12, pages 166-170, Dili, East Timor.

[DEMO 1998] DEMO, P. (1998). Educar pela Pesquisa. Autores Associados, Campinas, SP, $3^{a}$ edition.

[FRANCO 1998] FRANCO, S. (1998). O Construtivismo e a Educação. Mediação, Porto Alegre, RS.

[FRAWLEY 2000] FRAWLEY, W. (2000). Vygotsky e a Ciência Cognitiva: linguagem e integração das mentes social e computacional. Artmed.

[MAISONNETTE 1999] MAISONNETTE, R. (1999). A utilização dos recursos informatizados a partir de uma relação inventiva com a máquina: A robótica educativa. [online]. Disponível em http://www.proinfo.gov.br/upload/ biblioteca.cgd/192.pdf. Acessado em 28 de fevereiro de 2016.

[MARINS 1999] MARINS, L. (1999). Robôs enriquecem o currículo escolar e estimulam o cérebro. [online]. Disponível em http://www.gazetadopovo.com.br. Acessado em 28 de fevereiro de 2016.

[MICROSOFT 2015] MICROSOFT (2015). Eu posso programar. [online]. Disponível em https: / / www . eupossoprogramar. com/. Acessado em 28 de fevereiro de 2016.

[MIT Media Lab 2015] MIT Media Lab (2015). Scratch: Imagine, program, share. [online]. Disponível em https://scratch.mit.edu/. Acessado em 28 de fevereiro de 2016.

[MORIN 1996] MORIN, E. (1996). O problema epistemológico da complexidade. Lisboa: Publicações Europa-América.

[NOTÍCIAS DA CISCO. 2013] NOTÍCIAS DA CISCO. (2013). Estudo revela demanda crescente por profissionais de tecnologia no brasil, mas há falta de mão de obra qualificada. [online]. Disponível em http://globalnewsroom.cisco.com. Acessado em 06 de março de 2016.

[PEREIRA 2010] PEREIRA, G. (2010). O uso da robótica educacional no ensino fundamental: relatos de um experimento. Trabalho de Conclusão de Curso do Bacharelado em Ciência da Computação - Universidade Federal de Goiás, Campus de Catalão. Disponível em https://dcc. catalao.ufg.br/up/498/o/ Gabriela2010.pdf. Acessado em 05 de abril de 2015.

[RIBEIRO et al. 2011] RIBEIRO, P. C., MARTINS, C., and BERNARDINI, F. C. (2011). A robótica como ferramenta de apoio ao ensino de disciplinas de programação. In $22^{\circ}$ SBIE / $17^{\circ}$ WIE, 2011, Aracaju. 